\title{
Teste de Bender: Versão de Rastreio para a Avaliação da Maturidade Perceptomotora
}

\author{
Fernanda Otoni ${ }^{1}$, Fabián Javier Marín Rueda \\ Universidade São Francisco, Campinas, Brasil
}

\section{RESUMO}

Este estudo buscou evidências de validade de conteúdo e critério para a versão de rastreio do Bender - Sistema de Pontuação Gradual. Para isso, 693 crianças, ambos os sexos, com idades entre seis e 10 anos $(M=8,42 ; D P=1,38)$ reproduziram a cópia de três figuras. Os resultados indicaram que a Figura 3 apresentou alto nível de dificuldade, sendo necessário maior nível de maturidade perceptomotora para sua execução. As Figuras 7a e 7b, embora apresentem sobreposição, foram mais fáceis de serem copiadas. Verificou-se que os erros de distorções na reprodução das figuras tendem a diminuir gradualmente conforme aumenta a idade das crianças. Observou-se que as três figuras não apresentam funcionamento diferencial dos itens entre meninas e meninos, além disso também não foram encontradas diferenças de média de desempenho. Esses achados sugerem que a versão de rastreio é uma proposta promissora para ser utilizada na avaliação da maturidade perceptomotora.

Palavras-chave: teste de Bender; habilidade visomotora; avaliação psicológica; psicometria.

\section{ABSTRACT - Bender Test: Screening Version for Evaluation of Perceptive-Motor Maturity}

This study sought evidence of content and criteria validity for the screening version of the Bender - Gradual Scoring System. For this, 693 children of both sexes, between 6 and 10 years of age $(M=8.42 ; S D=1.38)$, reproduced three figures. The results indicated that Figure $3 \mathrm{had}$ a greater level of difficulty, with a greater level of perceptive-motor maturity required for its execution. Figures $7 \mathrm{a}$ and $7 \mathrm{~b}$, although overlapping, were easier to reproduce. It was found that the errors of distortions in the reproduction of figures tended to gradually decrease as the child's age increased. Another point observed was that the three figures did not present differential functioning for the items between girls and boys, nor were any mean performance differences found. These findings suggest that the screening version is a promising proposition for use in the evaluation of perceptive-motor maturity.

Keywords: Bender's test; visual-motor ability; psychological assessment; psychometry.

\section{RESUMEN - Test de Bender: Versión de Rastreo para la Evaluación de la Madurez Perceptomotora}

El estudio buscó evidencias de validez de contenido y criterio para la versión de rastreo del Bender - Sistema de Puntuación Gradual. Por esto, participaron 693 niños de ambos sexos con edades entre 6 y 10 años $(M=8,42 ; D P=1,38)$, que reprodujeron la copia de tres figuras. Los resultados indicaron que la Figura 3 presentó alto nivel de dificultad, siendo necesario un mayor nivel de madurez perceptiva para su ejecución. Las Figuras 7 a y 7 b, aunque presentaban superposición, fueron más fáciles de dibujar. Se verificó que los errores de distorsión en la reproducción de las figuras tienden a disminuir gradualmente según aumenta la edad de los niños. Otro punto observado fue que las tres figuras no presentan funcionamiento diferencial entre las niñas y niños, además tampoco se encontraron diferencias de promedio de rendimiento. Estos hallazgos sugieren que la versión de rastreo es una propuesta oportuna para ser usada en la evaluación de la madurez perceptiva.

Palabras clave: Test de Bender; habilidad visomotora; evaluación psicológica; psicometría.

O Teste Gestáltico Visomotor de Bender, ou simplesmente teste de Bender, é um instrumento composto por nove figuras que tem por princípio avaliar a maturidade perceptomotora (Bender, 1955; Sisto, Noronha, \& Santos, 2006; Rueda, Sousa, Santos, \& Noronha, 2016). Compreendida como a capacidade de perceber e integrar estímulos externos para expressar uma ação motora, essa habilidade tem sido relacionada à linguagem, a conceitos espaciais e temporais, à capacidade de organização, ao planejamento, à atenção, à memória e à coordenação motora (Bender, 1955; Koppitz, 1989; Sisto et al., 2006; Rueda et al., 2016; Sousa e Rueda, 2017). De acordo com Santos, Anache e Santana (2015) e Suehiro e Cardim (2016), o teste de Bender tem sido um dos instrumentos mais utilizados tanto no âmbito clínico quanto no escolar. Ao estar atrelada a diversas habilidades cognitivas subjacentes ao processo de aprendizagem, a maturidade perceptomotora tem se mostrado útil na predição

Endereço para correspondência: Avenida Engenheiro Antonio Francisco de Paula Sousa, 2601, 13044-502, Campinas, SP. Cel.: (35) 98891-2906. E-mail: fer_ottoni@hotmail.com

O presente trabalho foi realizado com apoio da Coordenação de Aperfeiçoamento de Pessoal de Nível Superior - Brasil (CAPES) - Código de Financiamento 001. 
de possíveis dificuldades de aprendizagem (Batista \& Gonçalves, 2016; Carvalho, Noronha, Pinto, \& Luca, 2012; Noronha, Santos, \& Rueda, 2013; Silva, Oliveira, \& Ciasca, 2017; Suehiro, Santos, \& Rueda, 2015). Crianças com dificuldades em reproduzir as figuras do teste de Bender tendem a apresentar prejuízos na leitura, escrita e baixo rendimento escolar (Batista \& Gonçalves, 2016; Carvalho et al., 2012; Noronha et al., 2013; Silva et al., 2017; Suehiro et al., 2015).

De acordo com Lauretta Bender (1955), precursora do teste, os erros que a criança pode cometer na reprodução das figuras podem ser provenientes de comprometimento intelectual ou de imaturidade perceptomotora. Ao analisar apenas de forma qualitativa o refinamento dos desenhos, Bender não criou um sistema de correção para o teste, e essa lacuna incentivou diversos autores a criarem diferentes sistemas de correção normatizados para avaliar o instrumento (Sisto et al., 2006). Dentre os diversos métodos de correção, um dos mais utilizados foi o sistema de Koppitz (1989), a autora considerou o caráter evolutivo da maturidade perceptomotora e criou uma tabela normativa que permitisse a inferência dos resultados em função da idade. Além disso, Koppitz seguiu os pressupostos de Bender de que o comportamento perceptomotor é uma habilidade que pode ser alcançada pelo uso de padrões complexos e propôs um sistema de correção a partir de quatro critérios de correção, a saber, integração, rotação, distorção de forma e perseveração (Kacero, 2005).

Embora tenha contribuído muito para o avanço dos estudos relacionados à maturidade perceptomotora e à aprendizagem, esse sistema apresentou pouquíssimas evidências que apontassem sua sensibilidade para avaliar o desempenho perceptomotor de crianças brasileiras. Como, por exemplo, a falta de evidências para predição do rendimento escolar, limitações para diagnósticos neuropsicológicos, divergências entre a captação da faixa etária de cada criança, diferenças entre os sexos, inapropriação para administração em crianças de 10 anos, entre outras (Bandeira \& Hutz, 1994; Bartholomeu, Rueda, \& Sisto, 2005). Os estudos de Sisto, Noronha e Santos (2004) e Sisto, Santos e Noronha (2004) ainda permitiram verificar que nem todos os critérios de correção eram válidos para avaliar a maturidade perceptomotora. Os autores observaram que apenas os critérios de integração e distorção da forma apresentavam resultados significativos para predizer os escores do teste Bender, no entanto, a baixa correlação com a idade indicava que o sistema de correção de Koppitz não era sensível para mensurar o aspecto maturacional do desenvolvimento infantil. Assim, Sisto et al. (2006) construíram o Bender - Sistema de Pontuação Gradual (B-SPG), e essa nova proposta de correção do teste de Bender tem sido o único método com parecer favorável pelo Conselho Federal de Psicologia (2018), disponível para ser utilizado em avaliação psicológica no Brasil.
No tocante ao B-SPG, a maturidade perceptomotora é avaliada por meio de padrões de diferentes graus de complexidade e princípios de organização, no qual pontuam-se os erros de distorção da forma, sendo esta compreendida pela falta de precisão nos aspectos estruturais do desenho (Sisto et al., 2006). Há um consenso entre os autores em afirmar que esse sistema também mantêm o aspecto desenvolvimental da maturidade perceptomotora, tal como concebido no teste originado por Bender (Pinto \& Noronha, 2010; Santos, Noronha, Rueda, \& Segovia, 2014; Sisto et al., 2006). Noronha et al. (2013) e Pinto e Noronha (2013), ao avaliarem crianças com idades entre seis e 10 anos, verificaram que conforme aumentava a idade, melhor seria a qualidade dos traçados na reprodução das figuras. Segundo Bender (1955), a percepção visual e a coordenação motora são formas primitivas da experiência humana. Desse modo, à medida que a criança amplia as experiências sensório-motoras, melhor é a capacidade de integrar as informações para construir uma imagem completa.

Por seguir os princípios da Gestalt na escolha das figuras do teste, a autora do instrumento (Bender, 1955) priorizou os desenhos constituídos por pontos ou laçadas (1, 2, 3 e 5), linhas retas e ângulos (A, 7a, 7b e 8) e linhas curvas (4, 6) (Kacero, 2005; Sisto et al., 2006). De acordo com Kacero (2005) e Rueda et al. (2016) essas figuras requerem noções de orientação espacial e temporal, planejamento e organização. Diversos autores afirmam que a maturidade perceptomotora apresenta um caráter desenvolvimental, sendo que a percepção visual e a coordenação motora embora tenha uma melhora gradual acontece de forma subjetiva para cada uma das crianças (Bender, 1955; Kacero, 2005; Sisto et al., 2006). Nessa perspectiva, Pinto e Noronha (2010) e Suehiro e Santos (2006) ao aplicarem as nove figuras do B-SPG em crianças com idades entre seis e 10 anos, verificaram que conforme aumentou a idade menores eram os erros de distorções cometidos pelas crianças.

Sisto, Santos e Noronha (2010), por sua vez, utilizaram a análise do funcionamento diferencial de item (DIF) para verificar se meninas ou meninos poderiam apresentar maior facilidade de endosso nas figuras do B-SPG. Os autores observaram que os meninos cometeram mais distorções na Figura 5, enquanto as meninas apresentaram o mesmo erro na Figura 6, no entanto uma figura sobrepõe o valor da outra e não causa prejuízos no processo de avaliação psicológica. Sisto et al. (2010) ainda verificaram que as figuras mais fáceis de serem reproduzidas foram a 7 a e $7 b$, enquanto a Figura 3 foi a mais difícil. Rueda e Jesuíno (2018) também encontraram DIF nas figuras do teste de Bender ao avaliar crianças com desenvolvimento típico e pessoas com diagnóstico de deficiência intelectual. Os resultados indicaram que crianças sem comprometimento intelectual tem maior facilidade na reprodução da Figura 6, enquanto o outro grupo é mais favorecido pelas Figuras 1 e 2 . Os autores avaliaram crianças com comprometimento intelectual de 
instituições de educação especial e sugerem que, por serem mais estimuladas, podem apresentar melhor maturidade perceptomotora.

Em relação às diferenças em razão do desempenho de médias entre meninas e meninos, Pinto e Noronha (2010) avaliaram a reprodução do desenho de 298 crianças $(52 \%$ meninos), com idades entre seis a 10 anos. De acordo com as autoras, embora os meninos tenham cometido mais erros de distorção $(M=9,58)$ que as meninas $(M=9,10)$, os resultados não foram significativos para afirmar que o teste tende a favorecer um dos sexos. Suehiro e Cardim (2016) analisaram os protocolos de 388 crianças baianas com idades entre seis e 10 anos e não encontraram diferenças significativas no desempenho entre os sexos. No entanto, puderam observar que os meninos com mais idade tiveram melhor desempenho. Em contrapartida ao estudo de Suehiro e Cardim (2016), Valderas et al. (2017) observaram que meninas de 10 anos apresentaram melhor maturidade perceptomotora que os meninos. Com base nos resultados desses estudos não existe um consenso entre em relação às diferenças de médias entre o sexo, e a maturidade perceptomotora parece estar mais relacionada ao desenvolvimento infantil e às habilidades cognitivas (Pinto \& Noronha, 2010; Suehiro \& Cardim, 2016; Valderas et al., 2017).

Em estudo recente, Rueda et al. (2016) verificaram se com um conjunto menor de figuras seria possível obter uma medida da maturidade perceptomotora consonante à proposta original do B-SPG. Assim, aplicaram todas as figuras do teste em 787 crianças das cidades de Minas Gerais, Piauí e Paraíba, com idades entre seis e 10 anos, sendo 56,5\% meninas. Após verificarem que os dados eram passíveis de fatoração $(\mathrm{KMO}=85$; $\left.\chi^{2}(45)=2152,370 ; p<0,001\right)$, os autores selecionaram aquelas com maior carga fatorial, propondo a versão de rastreio com as figuras $3,4,7 \mathrm{a}$ e $7 \mathrm{~b}$. Posteriormente, realizaram uma análise de regressão e verificaram que as figuras selecionadas explicaram $80 \%$ da variância do escore total do B-SPG. Os autores ainda investigaram se essa versão de rastreio do B-SPG seria capaz de diferenciar o desempenho das crianças em função da idade e verificaram que, conforme a idade das crianças aumentou, menores foram os erros de distorção $[\mathrm{F}(4,782)=72,16$; $p<0,001]$. A partir desses resultados, afirmaram que essas quatro figuras seriam capazes de mensurar a maturidade perceptomotora e, poderiam ser usadas para predizer possíveis dificuldades de aprendizagem.

Assim como Bender (1955), Rueda et al. (2016) também tiveram o cuidado de selecionar as figuras considerando os diferentes tipos de traçado, permitindo a compreensão de como a criança se orienta dentro de um determinado quadro de referência e como conseguem lidar com as relações espaciais. A Figura 3, por exemplo, é constituída por pontos. Na reprodução desse desenho, o desafio principal é atender simultaneamente as distâncias, posições, inclinações, proporções e coordenação com o ajuste dos ângulos. Já a figura 4 segue o princípio de continuidade, e na execução desse desenho a criança deve interromper bruscamente o movimento e mudar de direção. Essa ação implica uma construção sistemática do espaço com relação à outra figura. Finalmente, as figuras $7 \mathrm{a}$ e $7 \mathrm{~b}$ seguem o princípio da continuidade de formas, e as mudanças nas direções das partes e dos ângulos são habilidades perceptivas adquiridas mais tardiamente pelas crianças (Kacero, 2005).

Outras propostas de usar o Bender com um número menor de figuras já foram realizadas. Santucci e Pêcheux (1981), por exemplo, usaram apenas cinco cartões (Figuras A, 2, 4, 3, 7a e 7b, nessa ordem) e consideravam como critérios de correção a qualidade da forma, número de colunas ou círculos, conceito espacial, relação contiguidade-separação e junção ou separação das subpartes. Os autores buscavam discriminar as crianças que possuíam dificuldades de aprendizagem das crianças com comprometimento intelectual. Brannigan e Brunner (2002), por sua vez, criaram o Sistema de Classificação Qualitativa (SCQ) para avaliar o desempenho perceptomotor de crianças entre quatro e oito anos utilizando seis figuras (A, 1, 2, 4, 6 e 8). Ambas propostas justificaram que escolheram essas figuras por serem as mais apropriadas para avaliar a maturidade perceptomotora, não apresentando estudos psicométricos que embasassem essa afirmação. Para o primeiro sistema proposto com um menor número de figuras, não foram encontrados estudos que o tenham utilizado, já para o SCQ, alguns autores afirmam que esse sistema é sensível para predizer possíveis dificuldades de aprendizagem (Soto, 2014), no entanto, não é capaz de diferenciar o desempenho em função da idade (Soto, 2011).

Assim, ao compreender a importância de que todos os instrumentos psicológicos apresentem propriedades psicométricas adequadas (American Educational Research Association [AERA], American Psychological Association [APA], \& National Conuncilon Measurement in Education [NCME], 2014), esse estudo buscou evidências de validade de conteúdo e de critério, considerando a idade e o sexo, para a versão de rastreio do B-SPG. Além de verificar o nível de dificuldade das figuras, procurou-se investigar se a versão de rastreio é capaz de diferenciar o desempenho em razão da idade e, por fim, foi observado se existem diferenças de desempenho em função do sexo. Para que um instrumento seja considerado válido, é necessária uma gama de estudos que indiquem evidências de validade capazes de garantir que o teste é realmente adequado para determinado fim (AERA, APA, \& NCME, 2014). Sendo assim, ao confirmar o potencial da versão de rastreio do B-SPG em sinalizar dificuldades na maturidade perceptomotora, será possível contribuir para o planejamento de intervenções que possam minimizar o impacto das dificuldades de aprendizagem ainda nos estágios iniciais de escolarização. Ressalta-se que este é o primeiro estudo que busca evidências de validade para a versão de rastreio do B-SPG. 


\section{Método}

\section{Participantes}

Participaram do estudo 693 crianças com idades entre seis e 10 anos $(M=8,42 ; D P=1,38)$, estudantes de escolas públicas dos estados de São Paulo e Minas Gerais, matriculadas do primeiro ao quinto ano do ensino fundamental I de escolas públicas. As informações referentes à quantidade de crianças por idade e sexo serão apresentadas na Tabela 1 .

Tabela 1

Distribuição dos Participantes por Idade e Sexo

\begin{tabular}{cccccc}
\hline Idade & Meninas & $\%$ & Meninos & $\%$ & Total \\
\hline 6 & 40 & 53,3 & 35 & 46,7 & 75 \\
7 & 83 & 58,9 & 58 & 41,1 & 141 \\
8 & 57 & 50,4 & 56 & 49,6 & 113 \\
9 & 72 & 58,1 & 52 & 41,9 & 124 \\
10 & 113 & 47,1 & 127 & 52,9 & 240 \\
Total & 365 & 52,7 & 328 & 47,3 & 693 \\
\hline
\end{tabular}

\section{Instrumentos}

Versão de rastreio do Teste Gestáltico Visomotor de Bender: Sistema de Pontuação Gradual (BSPG). A versão de rastreio do Bender B-SPG (Rueda et al., 2016) é uma proposta que advém do sistema de pontuação gradual desenvolvido por Sisto, Santos e Noronha (2006) no qual utilizam nove figuras para avaliar a maturidade perceptomotora, por meio de figuras formadas por linhas contínuas ou pontos, curvas sinuosas ou ângulos. Na versão de rastreio, são administradas as figuras 3, 4, 7a e 7b, para a execução do teste, pede-se que a criança reproduza as figuras em uma folha em branco. A atribuição de notas é de zero a dois pontos, o critério de correção se baseia nos erros de distorção da forma, no qual a pontuação pode variar de zero a oito pontos, por pontuar apenas os erros, quanto menor for a pontuação, melhor é o desempenho no teste.

\section{Procedimentos}

Após a autorização das escolas para a coleta dos dados, a aprovação comitê de ética e a entrega do Termo de Consentimento Livre e Esclarecido (TCLE) assinado pelos pais, foram agendadas as aplicações coletivas. Foi disponibilizada uma sala de aula com retroprojetor, onde aproximadamente 20 alunos, juntamente com as professoras, eram encaminhados. Durante a aplicação do teste além da presença da pesquisadora e do professor, contou-se também com um psicólogo com conhecimento prévio do teste. Nessa sala, os alunos receberam uma folha sulfite e um lápis preto. As instruções para a execução da tarefa foram realizadas pela pesquisadora. Inicialmente foi realizado um rapport com as crianças a fim de minimizar a ansiedade e fazer com que elas se interessassem pela atividade, posteriormente foi pedido que escrevessem seu nome, idade e ano escolar. O tempo de aplicação foi de aproximadamente 30 minutos. A correção do protocolo da versão reduzida do B-SPG foi realizada conforme instruem as normas do manual.

\section{Análise de dados}

Por meio do programa Statistical Package for the Social Sciences 21 (SPSS) foi realizada a precisão entre avaliadores pela correlação de Pearson. As análises descritivas e inferenciais para uma melhor caracterização da amostra e para observar a média de desempenho dos participantes, assim como a análise de variância considerando a diferença entre as idades, a prova de post hoc por método de Tukey permitiu observar o agrupamento das faixas etárias. O teste $t$ de Student indicou se havia diferenças nas médias de desempenho em razão do sexo. Pelo programa Winsteps foi possível observar o nível de dificuldade das figuras, e se estas apresentavam funcionamento diferencial do item entre meninas e meninos.

\section{Resultados}

Com o objetivo de assegurar maior confiabilidade dos resultados, em relação à correção geral da pesquisadora, solicitou-se que uma juíza com expertise na correção do B-SPG avaliasse às cegas $20 \%(\mathrm{n}=140)$ dos protocolos. Embora tenham sido selecionados aleatoriamente, tomou-se o cuidado de contemplar todas as idades para correção. Os resultados obtidos por meio da correlação $r$ de Pearson indicaram que as correlações de cada uma das figuras separadamente (Figura 3: $r=0,92$; Figura 4: $r=0,89$; Figura 7a: $r=0,93$; e Figura 7b: $r=0,88$ ) foram estatisticamente significativas, positivas e $(p<0,001)$ de magnitude forte (Cohen, 1988), indicando haver uma ótima precisão dos resultados que serão apresentados posteriormente.

Iniciando as estatísticas inferências, verificou-se que a média geral dos escores das crianças foi de quatro 
pontos $(D P=2,04)$, sendo que poderia variar entre zero e oito pontos. Além disso, observou-se que apenas 2,59\% não cometeram nenhum erro de distorção, e somente 5,9\% alcançaram o máximo de pontos, apresentando o pior desempenho. Em relação a cada um dos desenhos reproduzidos pelas crianças, verificou-se que as Figuras $7 \mathrm{a}$ e $7 \mathrm{~b}(M=0,69 ; D P=0,77$ e $M=0,85 ; D P=0,78$, respectivamente) obtiveram maior frequência de acertos que as demais figuras, seguidas pela Figura 4, no qual, a frequência de melhor e pior desempenho foram próximas, sendo $25,5 \%$ e $24,8 \%$, respectivamente. A Figura 3, por sua vez, indicou que $63,3 \%$ não conseguiram reproduzi-la corretamente.

Na sequência, buscou-se verificar o nível de dificuldade de cada uma das figuras, para tal, análise utilizou-se o modelo de Rasch. As estatísticas descritivas sumarizadas referente a dificuldade das figuras e os índices de infit e outfit constam na Tabela 2.

Tabela 2

Estatísticas Descritivas Sumarizadas da Versão de Rastreio do B-SPG

\begin{tabular}{lcccc}
\multicolumn{1}{c}{ B-SPG rastreio } & Dificuldade & Infit & Outfit & Correlação Item-Total \\
\hline Figura 3 & $-1,46$ & 1,43 & 1,54 & 0,54 \\
Figura 4 & 0,02 & 1,01 & 1,14 & 0,61 \\
Figura 7a & 0,99 & 0,76 & 0,72 & 0,77 \\
Figura 7b & 0,45 & 0,75 & 0,77 & 0,76 \\
Média & & & & - \\
DP & 0,00 & 0,99 & 1,04 & - \\
\hline
\end{tabular}

Os resultados indicam que todas as figuras que compõem a versão de rastreio do B-SPG apresentaram índices de infit e outfit dentro do intervalo esperado 0,70 a 1,5 (Linacre, 2011). Embora o valor da Figura 3 tenha sido 1,54 para esse índice de ajuste, ele é marginalmente aceitável. Em relação ao nível de dificuldade, a Figura 3 indicou ser a mais fácil de errar, enquanto as Figuras $7 \mathrm{a}$ e $7 \mathrm{~b}$ foram as mais fáceis de acertar. No que tange à correlação item-total, foram obtidos coeficientes de magnitude moderada (Nakano, Primi, \& Nunes, 2015), indicando alta consistência entre as figuras para avaliar a maturidade perceptomotora.

Sendo a maturidade perceptomotora uma habilidade que apresenta um caráter evolutivo, recorreu-se à análise de variância (ANOVA) para verificar as diferenças em razão da idade, cujos resultados indicaram diferenças estatisticamente significativas na pontuação total da versão de rastreio do B-SPG entre as idades $[F(693$,
$2120)=61,55 ; p<0,005]$. A fim de identificar quais faixas etárias se diferenciavam entre si, os dados foram submetidos a prova post hoc por método de Tukey. Os grupos formados são apresentados na Tabela 3 .

Pode-se verificar que a prova de Tukey agrupou as idades em cinco conjuntos, indicando que existem diferenças de desempenho para cada uma das idades. Observou-se que a média de pontuação na versão de rastreio do B-SPG tende a diminuir gradualmente, ou seja, conforme a idade das crianças aumenta menores são os erros de distorção na reprodução das figuras e, consequentemente, melhor é a maturidade perceptomotora. Na sequência, realizou-se uma análise de DIF a fim de verificar se um dos sexos tendem a endossar mais um item em detrimento do outro, para tanto, utilizou-se o valor de DIF contraste superior a 0,64 como referência para atribuição de significância estatística (Linacre, 2011).

Tabela 3

Prova de Tukey em Função das Idades

\begin{tabular}{|c|c|c|c|c|c|c|}
\hline \multirow{2}{*}{ Idade } & \multirow{2}{*}{$\mathrm{N}$} & \multicolumn{5}{|c|}{ Subgrupo para alfa=0,005 } \\
\hline & & 1 & 2 & 3 & 4 & 5 \\
\hline 10 & 240 & 2,86 & & & & \\
\hline 9 & 124 & & 3,59 & & & \\
\hline 8 & 112 & & & 4,30 & & \\
\hline 7 & 141 & & & & 5,05 & \\
\hline 6 & 75 & & & & & 5,86 \\
\hline$p$ & & 1,00 & 1,00 & 1,00 & 1,00 & 1,00 \\
\hline
\end{tabular}


Tabela 4

DIF por Sexo e Valores de t por Item

\begin{tabular}{|c|c|c|c|c|c|c|c|}
\hline \multirow{2}{*}{ Item } & \multicolumn{2}{|l|}{ Meninas } & \multicolumn{2}{|l|}{ Meninos } & \multirow{2}{*}{ Contrast } & \multirow{2}{*}{ Joint SE } & \multirow{2}{*}{ Mantel } \\
\hline & DIF Measure & $\mathrm{SE}$ & DIF Measure & SE & & & \\
\hline Figura 3 & $-1,20$ & 0,09 & $-1,46$ & 0,09 & 0,26 & 0,13 & 0,053 \\
\hline Figura 4 & $-0,12$ & 0,11 & 0,11 & 0,10 & $-0,23$ & 0,15 & 0,245 \\
\hline Figura 7a & 0,90 & 0,11 & 0,96 & 0,10 & $-0,06$ & 0,14 & 0,466 \\
\hline Figura 7b & 0,40 & 0,10 & 0,40 & 0,09 & 0,00 & 0,14 & 0,658 \\
\hline
\end{tabular}

Os resultados apresentados na Tabela 4 indicaram que as figuras que compõem a versão de rastreio não apresentam funcionamento diferencial do item, pois eles não favorecem nenhum dos sexos. Em seguida, buscou-se verificar se existe diferença entre os grupos de meninas e meninos, considerando a idade. Para tal análise, recorreu-se ao teste $t$ de Student e ao $d$ de Cohen. Os resultados podem ser observados na Tabela 5 .

Tabela 5

Comparações das Diferenças entre Meninas e Meninos no Rastreio do B-SPG

\begin{tabular}{|c|c|c|c|c|c|c|c|}
\hline Idade & Sexo & $\mathrm{N}$ & $\mathrm{M}$ & $D P$ & $t$ & $p$ & $d$ \\
\hline \multirow{2}{*}{6 anos } & Meninas & 40 & 6,10 & 1,21 & \multirow{2}{*}{1,44} & \multirow{2}{*}{0,154} & \multirow{2}{*}{0,33} \\
\hline & Meninos & 35 & 5,60 & 1,77 & & & \\
\hline \multirow{2}{*}{7 anos } & Meninas & 83 & 5,12 & 1,94 & \multirow{2}{*}{0,45} & \multirow{2}{*}{0,655} & \multirow{2}{*}{0,08} \\
\hline & Meninos & 58 & 4,96 & 2,13 & & & \\
\hline \multirow{2}{*}{8 anos } & Meninas & 56 & 4,21 & 1,90 & \multirow{2}{*}{$-0,51$} & \multirow{2}{*}{0,531} & \multirow{2}{*}{0,09} \\
\hline & Meninos & 56 & 4,39 & 1,76 & & & \\
\hline \multirow{2}{*}{9 anos } & Meninas & 72 & 3,55 & 1,75 & \multirow{2}{*}{$-0,30$} & \multirow{2}{*}{0,764} & \multirow{2}{*}{0,05} \\
\hline & Meninos & 52 & 3,65 & 1,84 & & & \\
\hline \multirow{2}{*}{10 anos } & Meninas & 113 & 3,06 & 1,60 & \multirow{2}{*}{1,82} & \multirow{2}{*}{0,070} & \multirow{2}{*}{0,23} \\
\hline & Meninos & 127 & 2,68 & 1,61 & & & \\
\hline \multirow{2}{*}{ Total } & Meninas & 365 & 4,13 & 2,01 & \multirow{2}{*}{1,86} & \multirow{2}{*}{0,062} & \multirow{2}{*}{0,14} \\
\hline & Meninos & 328 & 3,83 & 2,07 & & & \\
\hline
\end{tabular}

De modo geral, os resultados permitem observar que os meninos de seis, sete e 10 anos cometeram menos erros de distorção que as meninas. Não foram encontradas diferenças estatisticamente significativas para nenhuma das idades. No entanto, do ponto de vista prático, o $d$ de Cohen indicou diferenças de efeito moderado (Cohen, 1988) para o grupo de crianças de seis e 10 anos.

\section{Discussão}

O objetivo deste estudo foi buscar evidências de validade para a versão de rastreio do B-SPG. Ao selecionar o conjunto de figuras que comporiam essa proposta que apresenta um número reduzido de figuras, Rueda et al. (2016) priorizam além daquelas que apresentaram maior carga fatorial, aquelas que seguem os diferentes princípios da Gestalt. De acordo com Bender (1938) e Kacero (2005), sabe-se que os diferentes ângulos e traçados das figuras permitem que a criança integre os estímulos e organize a sua percepção. Sendo assim, ao observar o nível de dificuldade das figuras que compõem a versão de rastreio do B-SPG, verificou-se que a Figura 3 apresenta a maior complexidade, ou seja, foi o item com maiores erros de distorção. Em contrapartida, pode-se observar que as crianças tencionaram em acertar mais as figuras $7 \mathrm{a}$ e $7 \mathrm{~b}$. Esses achados corroboram os estudos de Sisto et al. (2010) e Rueda e Jesuíno (2018), que embora tenham aplicado a versão original do B-SPG, com nove figuras, também encontraram resultados semelhantes.

Segundo Kacero (2005) e Rueda et al. (2016), a reprodução da Figura 3 requer a capacidade de considerar, simultaneamente, a distância, a posição, a inclinação, a proporção, o paralelismo, a obliquidade e o ajuste dos ângulos. Essas são habilidades adquiridas de acordo como o desenvolvimento perceptomotor da criança, por isso se justifica a dificuldade das crianças de seis, sete e oito anos 
reproduzirem essa figura. A Figura 7a e 7b, por sua vez, embora apresente sobreposição entre as figuras, é mais fácil de ser reproduzida porque as crianças precisam se atentar apenas aos ângulos do desenho. De modo geral, Rueda et al. (2016) sugerem que o conjunto de figuras que compõem a versão de rastreio apresentam estruturas mais complexas e requerem maior habilidade perceptomotora para a reprodução de desenhos sem distorções, e consequentemente com melhor qualidade.

Os resultados deste estudo ainda permitem afirmar que a versão de rastreio mantém o aspecto evolutivo da maturidade perceptomotora, pois crianças com idades menores tendem a apresentar maior dificuldade em manter os aspectos estruturais do desenho ao reproduzi-lo. Diferente de Rueda et al. (2016), que não encontraram diferenças de desempenho em crianças de seis e sete anos, a análise de variância do presente estudo apontou para diferenças significativas entre todas as idades. Esses resultados foram semelhantes aos de Noronha et al. (2013), que ao aplicarem as nove figuras do B-SPG, também não encontraram diferenças significativas entre o grupo de crianças com seis e sete anos. Pinto e Noronha (2013), por sua vez, aplicando as mesmas nove figuras e verificaram que o grupo de crianças com nove e 10 anos se diferenciaram das de sete e oito anos e, por fim, o grupo de crianças de seis anos se diferenciaram dos demais. Ainda que esses estudos tenham apresentado resultados diferentes da versão de rastreio, ressalta-se que a média da pontuação do B-SPG melhora gradualmente, sendo que os erros de distorções diminuem significativamente em crianças com nove e 10 anos de idade, sugerindo que estas apresentam maior facilidade em perceber os estímulos externos e representá-los em uma ação motora.

No que tange ao funcionamento diferencial das Figuras 3, 4, 7a e 7b em razão do favorecimento de meninas ou meninos, verificou-se que essas figuras não apresentam DIF (Linacre, 2011). Este resultado já era esperado, pois as figuras que Sisto et al. (2010) e Rueda e Jesuíno (2018) encontraram DIF não fazem parte da versão de rastreio do B-SPG. Os achados, do presente estudo indicam que assim como a proposta original do B-SPG, a versão de rastreio também se mostra confiável para avaliar a maturidade perceptomotora de crianças no que diz respeito ao sexo, uma vez que não apresentou maior possibilidade de endosso para um dos sexos. Ressalta-se que Rueda et al. (2016), ao escolher as figuram que comporiam a versão de rastreio do B-SPG, preocuparam-se não só em separar aquelas figuras que apresentavam maior precisão para medir o construto avaliado, como também tiveram o cuidado de contemplar todos os traçados propostos pelos princípios da Gestalt. Essas diferenças entre as figuras contribuem para a maior compreensão de quais são os tipos de erros de distorção cometidos pelas crianças.

Ao comparar a média de desempenho entre meninas e meninos considerando a idade, embora os meninos tenham apresentado melhor desempenho, não foram encontradas diferenças significativas. Entretanto, do ponto de vista prático, verificou-se que os grupos de seis e 10 anos apresentaram diferenças de efeito pequeno. Ainda foi possível observar que, com exceção do grupo de meninas com oito anos de idade, os meninos apresentaram pontuação mais elevada da maturidade perceptomotora. Valderas et al. (2017) verificaram que os meninos brasileiros com idades entre seis e nove anos tendem a cometer menos erros de distorção, no entanto as meninas de 10 anos apresentam melhor maturidade perceptomotora que os meninos. Suehiro e Cardim (2016), por sua vez, trabalharam com uma amostra de crianças com idades entre sete e 10 anos, e, embora tenham observado que os meninos de nove e 10 anos tiveram uma média melhor que as meninas, não encontraram diferenças significativas. De acordo com a literatura revisada, não existe um consenso entre as pesquisas em afirmar que meninos poderiam ter maior facilidade em reproduzir as figuras do B-SPG em detrimento das meninas (Batista \& Gonçalves, 2016; Valderas et al., 2017; Suehiro \& Cardim, 2016). No entanto, Batista e Gonçalves (2016), assim como no presente estudo, também verificaram que as meninas apresentaram menor maturidade perceptomotora, e justificaram que os meninos tendem a ter uma orientação espacial melhor que as meninas.

Oliveira et al. (2016), Sousa e Rueda (2017), Silva et al. (2017) sugerem que a maturidade perceptomotora tem sido mais atrelada a habilidades cognitivas como a atenção, a memória e a inteligência, sendo que a influência dessas variáveis estaria mais relacionada com a capacidade da criança cometer menores erros de distorções ao reproduzir as figuras do B-SPG. Sendo assim, pode-se considerar que a maturidade perceptomotora é progressiva e se aperfeiçoa por meio de treinamento e aprimoramento e, quando consolidada, pode se tornar facilitadora no processo de aprendizagem. Os resultados encontrados nesta pesquisa permitem concluir que a versão de rastreio do B-SPG pode ser um instrumento a ser incluso no processo de avaliação psicológica, pois além de avaliar a maturidade perceptomotora contribui para a avaliação clínica e em larga escala. Ressalta-se ainda que os níveis de dificuldade das figuras que compóem a versão de rastreio permitem observar diferentes detalhes sobre os traçados de cada criança, possibilitando maior visibilidade da integração dos estímulos e variabilidade de distorção da forma. Viabilizando o planejamento de intervenções que minimizem o impacto de possíveis dificuldades de aprendizagem.

Ainda que este trabalho contribua com a compreensão acerca do uso da versão de rastreio do B-SPG no contexto clínico algumas limitações devem ser indicadas, como, por exemplo, não ter sido utilizada uma amostra em diferentes regiões do Brasil com crianças estudantes de escolas públicas e particulares e com diferentes diagnósticos. Portanto, para nova agenda de pesquisa, 
sugere-se que estudos sanem essas limitações e, ainda, relacionem a versão de rastreio do B-SPG com instrumentos que avalie diferentes habilidades cognitivas e com o desempenho escolar. Estudos deste cunho tendem a contribuir para conhecer melhor a capacidade de cobertura e predição desse instrumento.

\section{Referências}

American Educational Research Association, American Psychological Association, \& National Council on Measurement in Education, (2014). Standards for educational and psychological testing. Washington, DC: American Educational Research Association.

Bandeira, R. D., \& Hutz, C. S. (1994). A contribuição dos testes DFH, Bender e Raven na predição do rendimento escolar na primeira série. Psicologia: Teoria e Pesquisa, 10(1), 59-72. Recuperado de http://bases.bireme.br/cgi-bin/wxislind.exe/iah/online/?IsisScript=iah/iah.xis\& $\mathrm{src}=$ google \&base $=$ LILACS\&lang $=$ p\&nextAction $=\operatorname{lnk} \&$ exprSearch $=150153 \&$ indexSearch $=$ ID

Batista, M. A., \& Gonçalves, V. A. (2016). Validade convergente entre os testes de Desenho de Silver (SDT) e Bender (B-SPG) por idade e sexo. Pecibes, 2, 44-51. Recuperado de http://www.seer.ufms.br/index.php/pecibes/index

Bartholomeu, D., Rueda, F. J. M., \& Sisto, F. F. (2005). Teste de Bender e dificuldades de aprendizagem: quão válido é o sistema de Koppitz? Avaliação Psicológica, 4(1), 13-21. Recuperado de http://pepsic.bvsalud.org/pdf/avp/v4n1/v4n1a03.pdf

Bender, L. (1955). Teste Gestáltico Visomotor (Trad. D. Carnelli). Buenos Aires: Paidos.

Brannigan, G. G., \& Brunner, N. A. (2002). Guide to the qualitative scoring system for the modified version of the Bender-Gestalt Test. Springfield: Publisher.

Carvalho, L., Noronha, A. P. P., Pinto, L. P., \& Luca, L. (2012). Maturidade perceptomotora e reconhecimento de palavras: Estudo correlacional entre o Bender-Sistema de Pontuação Gradual e o Teste de Reconhecimento de Palavras. Estudos de Psicologia, 29(3),371377. doi: 10.1590/S0103-166X2012000300007

Cohen, J. (1988). Statistical Power Analysis for the Behavioral Sciences. New York: AcademicPress.

Conselho Federal de Psicologia (2018). Resolução No 009, de 25 de abril de 2018. Brasília, DF: Conselho Federal de Psicologia. Recuperado de http://satepsi.cfp.org.br/docs/Resolu\%C3\%A7\%C3\%A3o-CFP-n\%C2\%BA-09-2018-com-anexo.pdf

Kacero, E. (2005). Test gestáltico visomotor de Bender: Una puesta en espacio de figuras. Buenos Aires: Lugar editorial

Koppitz, E. M. (1989). O teste guestáltico Bender para crianças. Porto Alegre: Artes Médicas.

Linacre, J. M. (2011). A user's guide to Winsteps, Program Manual 3.74.0. Chicago: Winsteps.com. Recuperado de http://www.winsteps.com/ index.htm

Nakano, T. C., Primi, R., \& Nunes, C. H. S. S. (2015). Análise de itens e Teoria de Resposta ao Item (TRI). Em C. S. Hutz, D. R. Bandeira, \& C. M. Trentini (Eds.), Psicometria [Psychometry] (pp. 97-124). Porto Alegre, RS: Artmed

Noronha, A. P. P., Rueda, F. M. J., \& Santos, A. A. A. (2013). Bender Gestalt Visual-Motor Test - Sistema de Pontuação Gradual(B-SPG): A studywithdifferent samples. Paidéia, 23(55), 179-185. doi:10.1590/1982-43272355201305

Noronha, A. P. P., Rueda, F. J. M., \& Santos, A. A. A. (2015). Diferenças regionais e as normas de interpretação do Teste de Bender-Sistema de Pontuação Gradual. Psicologia em Pesquisa, 9(1), 3-9. doi: 10.5327/Z1982-1247201500010002

Noronha, A. P. P., Santos, A. A. A., \& Rueda, F. J. M. (2013). Habilidad viso-motriz y deficiencia intelectual: Estudio de validez para el Bender-SPG. Acta Colombiana de Psicología, 16(2), 115-123. doi: 10.41718/ACP.2013.16.2.11

Oliveira, A. L. S., Kaiser, V., Azambuja, T. O., Mallmann, L. U., Lukrafka, J. L., \& Reppold, C. T. (2016). Visual-motor maturity and executive functions in schoolchildren. Paidéia, 26(64), 215-223. doi: 10.1590/1982-43272664201609

Pinto, L. P., \& Noronha, A. P. P. (2013). Bender - Sistema de Pontuação Gradual (B-SPG): Análise da Maturação Percepto-Motora de Crianças [Bender-Gradual Scoring System (B-SPG): Analysis of the maturation of visual-motor perception of children]. Interação em Psicologia, 17(3), 281-289. doi: 10.5380/psi.v17i3.29683

Pinto, L. P., \& Noronha, A. P. P. (2010). Maturidade perceptomotora e sua relação com idade e variáveis contextuais: Um estudo com o Bender (B-SPG). Encontro: Revista de Psicologia, 13(19), 145-155. Recuperado de http://www.pgsskroton.com.br/seer/index.php/renc/ article/view/2522

Rueda, F. J. M., \& Jesuíno, A. D. S. A. (2018). The Bender Gradual Scoring System in assessment of people with intellectual disabilities. Paideia, 28(8), 1-9. doi: 10.1590/1982-4327e28xx

Rueda, F. J. M., Sousa, V., Santos, A. A. A., \& Noronha, A. P. P. (2016). Bender - Sistema de Pontuação Gradual (B-SPG): Estudo para versão de rastreio. Psicologia: Teoria e Prática, 18(2), 117-128. doi: 10.15348/1908-6906

Santos, A. M., Anache, A. A., \& Santana, R. C. (2015). Overview of brazilian scientific production in psychological evaluation. Psico-USF, 20(3), 547-559. doi: 10.1590/1413-82712015200315

Santos, A. A. A., Noronha, A. P. P., Rueda, F. J. M., \& Segovia, J. L. (2014). Bender-Gradual scoring system: Performance of Brazilian and Peruvian children. Perceptual and Motor Skills: Physical Development and Measurement, 118(3), 89-908. doi:10.2466/03.10.PMS.118k25w7

Santucci, H., \& Pêcheux, M. G. (1981). Prova gráfica de organização perceptiva para crianças de 6 a 14 anos. Em R. Zazzo (Eds.), Manual para o exame psicológico da criança, 291-338. São Paulo: Editora Mestre Jou.

Silva, S. L. Z. R., Oliveira, M. C. C., \& Ciasca, S. M. (2017). Desempenho percepto-motor, psicomotor e intelectual de escolares com queixa de dificuldade de aprendizagem. Rev. Psicopedagogia, 34(103), 33-44. Recuperado de http://pepsic.bvsalud.org/scielo.php?script=sci_ar ttext\&pid $=$ S0103-84862017000100004

Sisto, F. F., Noronha, A. P., \& Santos, A. A. A. (2004). Distorção da forma no teste de Bender: Questionando seu critério de validade. Revista do Departamento de Psicologia da UFF, 16(2), 139-154. Recuperado de http://pepsic.bvsalud.org/scielo.php?script=sci_arttext\&pid=S1677$04712004000100002 \& \operatorname{lng}=\mathrm{pt} \& \mathrm{t} \operatorname{lng}=\mathrm{pt}$

Sisto, F. F., Noronha, A. P. P., \& Santos, A. A. A. (2006). Teste Gestáltico Visomotor de Bender: Sistema de pontuação gradual (B-SPG). Itatiba/ SP:Vetor Editora Psicopedagógica Ltda. 
Sisto, F. F., Santos, A. A. A., \& Noronha, A. P. (2004). Critério de Integração do Teste de Bender: Explorando Evidências de Validade. Avaliação Psicológica, 3(1), 13-20. Recuperado de http://pepsic.bvsalud.org/scielo.php?script=sci_arttext\&pid=S1677-04712004000100002

Sisto, F. F., Santos, A. A. A., \& Noronha, A. P. P. (2010). Diferential functioning of Bender visual-motor gestalts test items. Perceptual and Motor Skills, 110(1), 313-322. doi: 10.2466/PMS.110.1.313-322

Soto, C. M. (2011). Exploración de diferencias normativas en el Sistema de Calificación Cualitativa para el Test Gestáltico de Bender Modificado. Liberabit, 17(2), 199-209. Recuperado de http://www.scielo.org.pe/scielo.php?script=sci_arttext\&pid $=$ S1729-48272011000200009

Soto, C. M. (2014). Validez incremental del Test Gestáltico de Bender Modificado, en niños que inician el primer grado. Avances en Psicología Latinoamericana, 32(2), 275-286. doi: 10.12804/ap132.2.2014.07

Sousa, V., \& Rueda, F. J. M. (2017). The relationship between perceptual motor skills and attention. Paidéia, 27(66), 24-32. doi:10.1590/1982432727662017046201704

Suehiro, A. C. B., \& Cardim, N. A. (2016). Bender-sistema de pontuação gradual: Uma comparação por idade, ano e sexo em crianças baianas. Avaliação Psicológica, 15(2), 257-264. doi: 10.15689/ap.2016.1502.14

Suehiro, A. C. B., Santos, A. A. A., \& Rueda, F. J. M. (2015). Desenvolvimento perceptomotor e escrita em crianças do ensino fundamental. Psicologia Escolar e Educacional, 19(2), 369-376. doi: 10.1590/2175-3539/2015/0192861

Valderas, R. V., Segovia, J. L., Moran, M. O., Alfaro, J. C., Vargas, G. S., Santos, A. A. A., Sisto, F. F., \& Noronha, A. P. P. (2017). La habilidad visomotora en niños escolares: Un estúdio transcultural Perú-Brasil. Psique Mag., 6(1), 73-89. Recuperado de http://ojs.ucvlima.edu. pe/index.php/psiquemag/article/view/174/92

\section{Sobre os autores}

Fernanda Otoni é psicóloga formada pela Unifenas/Alfenas, mestre e doutoranda em Psicologia pelo Programa de Pós-Graduação Stricto Sensu da Universidade São Francisco.

Fabían Javier Marín Rueda é psicólogo e doutor em Psicologia pelo Programa de Pós-Graduação Stricto Sensu da Universidade São Francisco. Atualmente é professor e coordenador no Programa de Pós-Graduação Stricto Sensu da Universidade São Francisco no campus Campinas - SP. 